\title{
ANÁLISE CLIMATOLÓGICA DA PRECIPITAÇÃO E DO TRANSPORTE DE UMIDADE NA REGIÃO DA ZCAS ATRAVÉS DA NOVA GERAÇÃO DE REANÁLISES
}

\author{
MARIO FRANCISCO LEAL DE QUADRO ${ }^{1,2}$, MARIA ASSUNÇÃO FAUS DA SILVA DIAS ${ }^{2}$, DIRCEU \\ LUIS HERDIES ${ }^{3}$, LUIS GUSTAVO GONÇALVES DE GONÇALVES ${ }^{3}$, \\ ${ }^{1}$ Instituto Federal de Educação, Ciência e Tecnologia de Santa Catarina (IFSC), Florianópolis, SC, Brasil \\ ${ }^{2}$ Universidade de São Paulo, Instituto de Astronomia, Geofísica e Ciências Atmosféricas (IAG/USP), \\ Departamento de Meteorologia, São Paulo, SP, Brasil \\ ${ }^{3}$ Instituto Nacional de Pesquisas Espaciais , Centro de Previsão de Tempo e Estudos Climáticos (INPE/ \\ CPTEC), Cachoeira Paulista, SP, Brasil
}

mquadro@ifsc.edu.br,mafdsdia@model.iag.usp.br,dirceu.herdies@cptec.inpe.br, gustavo.goncalves@cptec.inpe.br

Recebido Janeiro de 2011 - Aceito Novembro de 2011

\begin{abstract}
RESUMO
O conhecimento do desempenho das reanálises e dos erros associados a elas apresenta um papel fundamental na compreensão dos processos físicos que ocorrem na atmosfera. Este trabalho tem por objetivo documentar as principais características da precipitação associada à Zona de Convergência do Atlântico Sul (ZCAS), baseado em seis conjuntos de reanálises atmosféricas (MERRA, ERAInterim, ERA-40, NCEP 1, NCEP 2 e NCEP CFSR) e cinco conjuntos de produtos observados de precipitação (SALDAS, CPC, CMAP, GPCP e GLDAS). Através destes dados também foi analisado o transporte de umidade sobre a região da ZCAS, para os anos de 1979 a 2007. Em resumo, este trabalho evidencia o avanço das novas reanálises na tentativa de representar de forma mais adequada a variável precipitação acumulada. Os diagramas de Taylor mostram que os produtos de precipitação estão bem correlacionados com o ponto de referência (CPC), com coeficientes entre 0,6 e 0,9 . Somente a reanálise do NCEP CFSR possui correlações próximas as dos produtos de precipitação. Os conjuntos mais antigos de reanálises apresentam correlações menores, abaixo de 0,6. O Oceano Atlântico é a fonte principal do fluxo de umidade para a direção da ZCAS, que diminui na direção do continente. $\mathrm{Na}$ Região SE do Brasil, a topografia tem um papel importante para a convergência de umidade. Já na parte noroeste da ZCAS, este fator deve estar associado a processos termodinâmicos.
\end{abstract}

Palavras-chave: reanálise, ZCAS, precipitação

ABSTRACT: CLIMATOLOGICAL ANALYSIS OF THE PRECIPITATION AND UMIDITY TRANSPORT ON THE SACZ REGION USING THE NEW GENERATION OF REANALYSIS

The knowledge of the reanalysis performance and the systematic errors associated with them is extremely important in the acknowledgement of the physical processes occurring in the atmosphere. The goal of this study is to document the main characteristics of precipitation over the South Atlantic Convergence Zone (SACZ), based on six atmospheric reanalysis (MERRA, ERA-Interim, ERA-40, NCEP 1, NCEP 2 and NCEP CFSR) and five observed precipitation data products (SALDAS, CPC, CMAP, GPCP and GLDAS). Using the aforementioned available datasets the transport of moisture over SACZ was evaluated for the period of 1979 through 2007. In summary, the new generations of reanalyses show an improvement in the representation of the rainfall patterns and their magnitude. Taylor diagrams show that the observational products tend to be tightly grouped and close to the reference (CPC) point, whereas most of reanalyses show correlation coefficients below 0.6. NCEP CFSR reanalysis however, presented higher correlation coefficients relative to the others. Atlantic Ocean is the main moisture flux source to SACZ, which decreases towards the continent. Topography has an important role in moisture convergence over SE Region of Brazil. Over Amazon region, the convergence must be associated with thermodynamic processes.

Keywords: reanalysis, SACZ, precipitation 


\section{INTRODUÇÃO}

Durante os meses de verão do Hemisfério Sul, AZCAS é um fenômeno meteorológico que exerce um papel preponderante no regime de chuvas na região onde atua, acarretando altos índices pluviométricos na América do Sul. Este fenômeno é caracterizado pela persistência de uma banda de nebulosidade orientada no sentido noroeste-sudeste (NW-SE), que se estende desde o centro sul da Amazônia, regiões Centro-Oeste e Sudeste, centro sul da Bahia, norte do Estado do Paraná e estendendo-se em direção ao Oceano Atlântico sudoeste (Ferreira et al., 2004). Uma das principais consequências da atuação da ZCAS é a ocorrência dos altos índices pluviométricos, principalmente no final da primavera e nos meses de verão, nas regiões afetadas (Grimm, 2011).

Na década de 1980 e início dos anos 1990, os estudos concentraram-se no diagnóstico do sistema, através de sua caracterização física, buscando uma assinatura do fenômeno (Satyamurti e Rao, 1988; Silva Dias et al., 1991). Na época os trabalhos já apontavam a ZCAS ligada a fatores como: a interação de vórtices ciclônicos de altos níveis de escala subsinótica com sistemas frontais na região da ZCAS (Nobre, 1988), uma resposta a passagens de perturbações atmosféricas moduladas pela Oscilação de 30/60 dias (Casarin e Kousky, 1986) e explosões convectivas sobre o Brasil Central e sul da Amazônia, responsáveis pela geração da Zona de Convergência em baixos níveis (Figueroa e Nobre, 1990). Nesse período, também foram sugeridas possíveis influências remotas através de fenômenos meteorológicos ou oceânicos (Grimm e Silva Dias, 1995; Jones e Horel, 1990; Marton, 2000). Nos últimos anos também são realizados trabalhos de modelagem numérica para simulação da ZCAS nas suas componentes atmosférica e oceânica (Pezzi e Cavalcanti, 2000; Mendonça (2005); Chaves e Satyamurty, 2006) e estudos da variabilidade da ZCAS nas suas diversas escalas espaciais e temporais (Carvalho et al., 2004; Ferreira et. al.,2004; Grimm e Zilli, 2009).

Um dos fatores importantes para a localização da ZCAS é a topografia no centro-leste do Brasil, conforme demonstrado por Grimm et al. (2007). Esta topografia intensifica a precipitação, contribuindo para a manutenção da convecção na região através da geração de circulação ciclônica e convergência em baixos níveis (mecanismo CISK, instabilidade condicional de segunda ordem). A manutenção da ZCAS deve-se à contribuição da umidade que pode ser gerada localmente, pela evapotranspiração, ou transportada de outras áreas para a região da ZCAS. Herdies et al. (2002), analisando o transporte de umidade entre os trópicos e subtrópicos, associado ao padrão bimodal de ZCAS-NZCAS (Não ZCAS), concluíram que este transporte ocorre por dois caminhos principais, um associado à presença da ZCAS e outro ao Jato de Baixos
Níveis (JBN). Especificamente com relação à ZCAS, Jones e Carvalho (2002) mostram que variações intrasazonais nos ventos de baixos níveis sobre Amazônia modulam o SMAS e que os casos mais intensos de ZCAS estão relacionados a regimes de ventos de oeste, associados à fase ativa do SMAS. Drumond et. al. (2008), analisando o transporte de umidade médio pelo período de 10 dias, mostram uma forte captação de umidade sobre a costa leste do Brasil, na vizinhança da ZCAS e sugerem que a região sul do Atlântico Tropical é uma fonte de umidade para a ZCAS.

Nesse sentido, o Sistema de Monção da América do Sul (SMAS) tem papel importante para o transporte de umidade para a região Central da América do Sul e a configuração da ZCAS (Grimm, 2011). Nogués-Peagle et al. (2002) mostram que o SMAS desenvolve-se durante a primavera (setembronovembro), associado à migração da convecção para a região central da Amazônia, atinge o pico máximo durante o verão, quando ocorre um máximo de precipitação sobre a região central da Amazônia e a Região SE do Brasil e tem sua fase de dissipação no início do outono, em torno do mês de março, quando o máximo da convecção retorna para o extremo norte da AS diminuindo o gradiente de temperatura leste-oeste (continente-oceano).

Um sistema de monção é definido pela inversão sazonal do vento nos baixos níveis da troposfera em resposta ao contraste térmico entre regiões continentais e oceânicas adjacentes. Apesar da inversão sazonal do vento no SMAS não ser bem definida como na monção clássica, Zhou e Lau (1998), mostram que ao se subtrair a média anual do vento, a inversão sazonal característica do regime de monção é evidente, associada ao forte aquecimento diabático centrado no platô do Altiplano Boliviano. Recentemente, a descoberta de relação entre anomalias de precipitação na primavera e no verão no centro-leste da América do Sul (e também no sudeste), mostra a importância da interação superfície-atmosfera durante estas estações, envolvendo anomalias de umidade do solo e de temperatura junto à superfície no centro-leste do Brasil, anomalias de TSM junto à costa sudeste e a topografia no centroleste (Grimm et al., 2007; Grimm e Zilli, 2009).

O SMAS tem relação direta com a ZCAS, modulando o ciclo sazonal da precipitação sobre a América do Sul tropical em distintas estações seca e chuvosa em uma região compreendida entre o equador e 25S (Silva, 2009). Gan et al. (2004) mostra que $50 \%$ da precipitação anual sobre a América do Sul tropical e subtropical ocorre nos meses de verão austral (dezembro a fevereiro) e cerca de $90 \%$ durante os meses de outubro a abril. Marengo (2005), analisando a variabilidade temporal e espacial do balanço de umidade na região da bacia amazônica e arredores, mostrou que os períodos de primavera e verão apresentam forte convergência de umidade encontrada ao longo da ZCAS. 
Uma das ferramentas que podem ser utilizadas no estudo da ZCAS são as reanálises. Um sistema de reanálise consiste da combinação de um modelo de previsão (como background) e um sistema de assimilação de dados. Como as observações possuem uma distribuição irregular no espaço e no tempo, a assimilação combina essas informações disponíveis com o modelo de previsão para gerar uma nova análise. A primeira reanálise produzida foi nos anos de 1990 por Kalnay et al. (1996) junto ao National Centers for Environmental PredictionNational Center for Atmospheric Research (NCEP/NCAR). Na mesma década, outros conjuntos de reanálises foram gerados. O European Center for Medium Range Forecasting (ECMWF) produziu a reanálise ERA15 (Gibson et al. 1997), limitada a um curto período de tempo (1979-1993). O Center for OceanLand-Atmosphere Studies (COLA) também produziu uma reanálise curta cobrindo o período de maio de 1982 a novembro de1983 (Paolino et al. 1995). O Goddard Space Flight Center (GSFC/NASA), por sua vez, também produziu uma reanálise de 1980 a 1994 (Schubert et al. 1997). Nos anos 2000, a Japan Meteorological Agency (JMA) gerou o JRA25 (Onogi et al. 2007) e o ECMWF duas reanálises subseqüentes: o ERA40 (Uppala et al. 2005) e o ERA-Interim (Simmons et. al., 2007). Mais recentemente, o GSFC produziu a reanálise MERRA (Bosilovich, 2008), enquanto que o NCEP desenvolveu o Climate Forecast System Reanalysis (CFSR) (Saha et al., 2010). Em relação às reanálises anteriores (NCEP 1 e NCEP 2), Silva et al. (2011) ressalta as três principais diferenças do CFSR: 1) alta resolução vertical e horizontal, 2) as simulações são realizadas com um sistema acoplado atmosfera-oceano-mar gelo-terra, e 3) medidas históricas das radiâncias dos satélites são assimiladas.

As observações assimiladas no sistema de reanálise, e as parametrizações do modelo podem afetar previsão de precipitação subseqüente, devido à complexa interação entre o modelo e as observações (Kalnay et al., 1996). Além disso, é conhecido que a precipitação é um diagnóstico meteorológico crítico que não somente é bastante sensível ao sistema de observação e a física do modelo, como também reflete os efeitos da circulação geral da atmosfera (Bosilovich, 2008). Janowiak et al. (1998) testaram a reanálise do NCEP-NCAR com diversas análises estatísticas em relação aos dados do Global Precipitation Climatology Project (GPCP), obtidos a partir de dados de precipitação e estimativas com dados de satélites. Os resultados indicam que este sistema de reanálise apresenta um viés significativo.

O objetivo principal desse trabalho é de documentar as características principais do regime pluviométrico sobre a região da ZCAS nos meses de verão e inverno do período de 1979 a 2007. O estudo é feito a partir da comparação da precipitação obtida de seis conjuntos de reanálises atmosféricas (MERRA, ERA-Interim, ERA 40, NCEP 1, NCEP 2 e NCEP CFSR) e cinco conjuntos de produtos de precipitação (SALDAS
(Gonçalves et al., 2009), CPC (Xie et al. 2010), CMAP (Xie e Arkin, 1997), GPCP (Adler et al., 2003) e GLDAS (Rodell et al., 2004)). Uma análise adicional é apresentada visando investigar o fornecimento de umidade especialmente sobre a região da ZCAS.

\section{DADOS E METODOLOGIA}

A Figura 1 apresenta a área selecionada para obtenção dos cálculos estatísticos que visam analisar as características do regime de chuvas e do transporte de umidade para a região analisada, que compreende parte da região de atuação da ZCAS. A região selecionada foi baseada na Figura 1 do trabalho de Grimm (2011), que mostra o ciclo anual de precipitação sobre a América do Sul, calculado no período de 1950 a 2005.

Nesse estudo, seis conjuntos de reanálises são avaliados durante o período de 1979 a 2007. O NCEP disponibiliza dois conjuntos de reanálises denominados NCEP 1 e NCEP 2 (Kanamitsu et al. 2002) e o ECMWF fornece o conjunto ERA-40. Também é incluída neste trabalho a nova geração de conjuntos de reanálises ERA-Interim do ECMWF, MERRA produzido pelo GSFC e o mais recente conjunto de dados NCEP CFSR. O objetivo desta avaliação é o de analisar o desempenho da nova geração de reanálises, que possuem uma resolução espacial maior e modelos oceano-atmosfera-terra acoplados, como no caso do CFSR em relação aos produtos anteriores do NCEP (Silva et al., 2011).

Com relação a esta nova geração dos produtos de reanálises, o CFSR do NCEP foi desenvolvido para simular a condição do domínio acoplado do sistema oceano-atmosferaterra e gelo da superfície do mar, com alta resolução para o período de 1979 a janeiro de 2010. O modelo atmosférico global possui resolução de aproximadamente 38 km (T382)

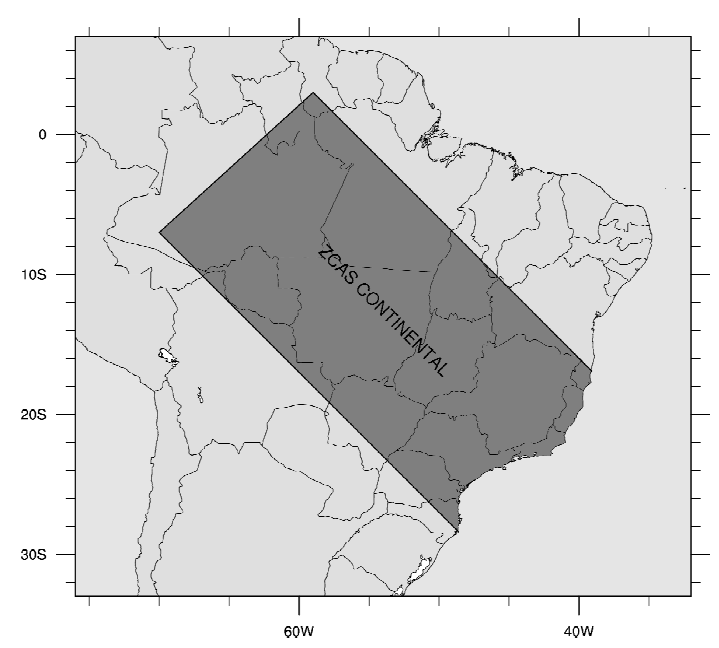

Figura 1 - Região selecionada para a análise deste estudo. As linhas sólidas mostram o limite dos pontos de grade (1.0 X 1.0). 
com 64 níveis na vertical. Na componente oceânica é utilizado o modelo MOM (Modular Ocean Model) versão 4p0d (Griffies et al. 2004). Este modelo possui espaçamento de grade de $0.25^{\circ}$ entre as latitudes de $10 \mathrm{~S}$ e $10 \mathrm{~N}$, aumentando gradualmente na direção dos pólos até $0.5^{\circ}$ nas latitudes de $30 \mathrm{~N}$ e $30 \mathrm{~S}$. Na vertical o MOM possui 40 níveis, contendo 27 camadas acima de 400, até uma profundidade de 4737 metros. O modelo de superfície possui quatro camadas de solo e o modelo de gelo marinho três camadas. Ressalta-se também, que o modelo atmosférico assimila as variações de $\mathrm{CO}_{2}$ desde 1979 até o presente, conjuntamente com as variações nos aerossóis, outros gases traço e solares. A reanálise MERRA possui uma alta resolução espacial $\left(0.5^{\circ}\right.$ de Latitude por $0.67^{\circ}$ de Longitude) e está disponível a cada 3 horas, a partir de 1979. Ela foi gerada utilizando o sistema global de assimilação de dados da NASA (National Aeronautics and Space Administration), com ênfase na estimativa do ciclo hidrológico e uma vasta variedade de informações meteorológicas nas escalas de tempo e clima. Já a reanálise do ERA-Interim faz parte da segunda geração dos produtos recentemente disponibilizados pelo ECMWF, desenvolvida para substituir a reanálise ERA-40 (disponível de 1979 a 2002). Esse conjunto de dados possui um espaçamento de grade de $1.5^{\circ}$ de Latitude/Longitude, que compreende o período de 1987 até o presente.

Estas reanálises são comparadas com cinco produtos de dados de precipitação baseados em observações, que são combinadas com estimativas de precipitação geradas a partir de dados de satélite. O primeiro conjunto de dados é a análise de dados globais registrados diariamente (denominado aqui de (PC), e produzidos para uma resolução espacial de $0.5^{\circ}$ de latitude / longitude, a partir de interpolação e controle de qualidade dos dados de precipitação reportados por aproximadamente 30.000 estações através do sistema Global Telecommunication System (GTS), além de outras fontes de dados. Neste estudo o CPC é considerado como conjunto de referência. No entanto, como o CPC é baseado principalmente em dados de precipitação observada interpolados pelo método de Interpolação Ótima (Gandim, 1965), e a região de interesse (Brasil Central) possuir poucos registros históricos de dados pluviométricos, outros conjuntos de dados de precipitação são utilizados na avaliação, como o Global Precipitation Climatology Project (GPCP), o Climate Prediction Center Merged Analysis of Precipitation (CMAP), o Global Land Data Assimilation System (GLDAS) e o South America Land Data Assimilation System (SALDAS). Para analisar o padrão espacial da precipitação, são calculadas as médias mensais de precipitação de cada conjunto de reanálise, assim como, dos produtos de precipitação observada. Ressalta-se que todos os conjuntos de dados são interpolados para a grade $1.0^{\circ} \times 1.0^{\circ}$ de resolução espacial, visando calcular as diferenças entre as médias mensais (viés). Também é analisado o desempenho das reanálises em produzir o padrão espacial da distribuição sazonal da precipitação através de histogramas e diagramas de Taylor (Taylor 2001). Neste último tipo de análise, a habilidade (skill) da reanálise é reproduzida pela distribuição anual da precipitação nas regiões pré-definidas. O desvio padrão normalizado (STD) aumenta com a distância radial em relação à origem. Todos os STD são normalizados em relação ao CPC, de forma que o valor 1.0 é o próprio valor de referência (REF).

Através dos dados acima mencionados, em conjunto com informações troposféricas (temperatura, vento, umidade específica) e de superfície (evaporação), foi avaliado para o período de 1979 a 2007 o fornecimento de umidade sobre a América do Sul e, especialmente para a região da ZCAS, somente para as reanálises da nova geração MERRA e NCEP CFSR para analisar o comportamento dessas novas séries de dados sobre o continente da América do Sul. O transporte de umidade através do cálculo da divergência do fluxo de umidade integrado na vertical é apresentado pela Equação 1. A unidade é mm.dia ${ }^{-1}$ e os valores negativos indicam convergência de umidade. Esta variável meteorológica é avaliada conjuntamente com a precipitação e a evaporação de cada reanálise, visando avaliar a capacidade de cada reanálise em simular a ZCAS durante do verão austral.

$$
D I V F U=\nabla \cdot\left(q \vec{V}_{H}\right)
$$

onde $q \vec{V}_{H}$ é o fluxo de umidade integrado na coluna atmosférica em Kg.m ${ }^{-1} \mathrm{~s}^{-1}$, q é a umidade específica do are $\overrightarrow{V_{H}}=V x \vec{i}+V y \vec{j}$ é o vento horizontal nas componentes zonal $\left(\mathrm{V}_{x}\right)$ e meridional $\left(\mathrm{V}_{y}\right)$.

\section{RESULTADOS}

A Figura 2 apresenta a precipitação climatológica para cada um dos produtos de precipitação (Figura 2a) e uma comparação com as reanálises (Figura 2b), para a região de estudo (Figura 1). Na região da ZCAS o ciclo anual dos produtos de observação é bastante similar (Figura 2a). As maiores diferenças são observadas justamente nos meses de atuação da ZCAS (outubro a março), sendo que os conjuntos GLDAS e GPCP apresentam os maiores valores médios de precipitação, em torno de $9 \mathrm{~mm} /$ dia entre janeiro e fevereiro, e os restantes (CPC, SALDAS e CMAP) em torno de $8 \mathrm{~mm} /$ dia neste período. As reanálises geradas pelo NCEP (NCEP 1, NCEP 2) e CFSR apresentam uma boa representação da precipitação observada (Figura 2b). Em uma análise mais detalhada, verifica-se que o CFSR, em relação ao reanálises anteriores, apresenta um atraso no início da estação chuvosa do SMAS e maiores índices de precipitação na região da ZCAS. Este resultado está de acordo com o de Silva et al. (2011), que também mostra um viés seco 
do CFSR durante o início da estação chuvosa do SAMS e úmido durante as fases de pico e decaimento do SAMS. Este fato pode estar relacionado com viés na umidade do solo e/ou evapotranspiração, e os efeitos que estes têm sobre o início da precipitação no modelo. Com relação às reanálises do ECMWF, enquanto a reanálise ERA-40 mostra um viés negativo significativo, o ERA-Interim superestima a precipitação dos produtos de precipitação na região da ZCAS. Segundo Simmons et al. (2007), o maior avanço do ERA-Interim reside no sistema de assimilação de dados que usa o 4D-Var (com ciclos de 6 e 12 horas de assimilação), que melhora substancialmente o desempenho da previsão, especialmente no Hemisfério Sul. A reanálise MERRA, assim como o ERA-40, também mostra um viés negativo significativo de precipitação, em torno de 3 a $4 \mathrm{~mm} /$ dia menor que os produtos de precipitação observada na região de estudo.

A Figura 3 apresenta o mapa de diferença entre a precipitação climatológica sazonal das reanálises e o CPC, para o período de verão austral (DJF). Em geral, as reanálises representam a distribuição da precipitação média anual sobre a América do Sul e, conseqüentemente, os regimes de precipitação associados aos sistemas meteorológicos atuantes (figura não mostrada). No entanto, em algumas regiões específicas é observado um viés de precipitação. Na parte norte do continente, na região da Zona de Convergência Intertropical (ZCIT), as reanálises apresentam geralmente chuvas acima do normal. Lin (2007), analisando vários Modelos de Circulação Geral Oceânicos e Atmosféricos, também encontrou este resultado, com a formação de uma dupla ZCIT e intensa precipitação sobre grande parte dos trópicos, que causa o fortalecimento demasiado dos ventos alísios, fluxo de calor latente à superfície excessivo e insuficiente fluxo de radiação de onda curta à superfície. Este excesso de umidade é transportado para a região central do continente da AS, através dos ventos alísios, influenciando na precipitação destas regiões. Por exemplo, sobre a região do Brasil Central, as reanálises MERRA e ERA-Interim apresentam sinais opostos em relação à climatologia do $\mathrm{CPC}$, onde o ERAInterim (Figura 3b) mostra chuvas acima do normal e o MERRA, assim também como o ERA-40, apresentam deficiência de precipitação (Figura 3a e 3c, respectivamente). Na faixa leste do Brasil tanto o NCEP 1 (Figura 3d) como o NCEP 2 (Figura 3e) mostram um viés positivo de precipitação, mais significativo sobre o NE do Brasil, que é reduzido pela nova reanálise CFSR. Em geral, os melhores resultados são apresentados pela reanálise CFSR (Figura 3e), particularmente sobre a região dos Andes. De acordo com Silva et al. (2011) essa consequência pode ser atribuída ao fato desta reanálise não assimilar a precipitação diretamente, mas sim ingerir os dados do GLDAS a partir das propriedades de superfície.

Nos meses de inverno do Hemisfério Sul (Figura 4), excetuando a reanálise do ERA-Interim na região tropical da AS, verifica-se uma redução das diferenças entre a precipitação das reanálises e do CPC. Esta redução é esperada, tendo em vista que a maior parte do continente tem pouca precipitação nesse período. Assim como no verão, o CFSR também apresenta as menores diferenças com relação à precipitação climatológica do CPC.

Especificamente na região de atuação da ZACS, a Figura 5 apresenta os histogramas da diferença entre a precipitação média no verão do Hemisfério Sul das reanálises avaliadas e a climatologia do CPC. É interessante observar as altas frequências de diferenças negativas do MERRA e ERA-40 (Figura 5a e 5c, respectivamente) e positivas do ERA-Interim (Figura $5 \mathrm{~b}$ ) associadas aos padrões espaciais verificados na Figura 3. A reanálise do NCEP CFSR foi a que melhor representou uma distribuição normal centrada entre $-0,5$ e $0,5 \mathrm{~mm} /$ dia, enquanto que as reanálises do NCEP 1 e NCEP 2 mostram uma distribuição das frequências semelhantes (com
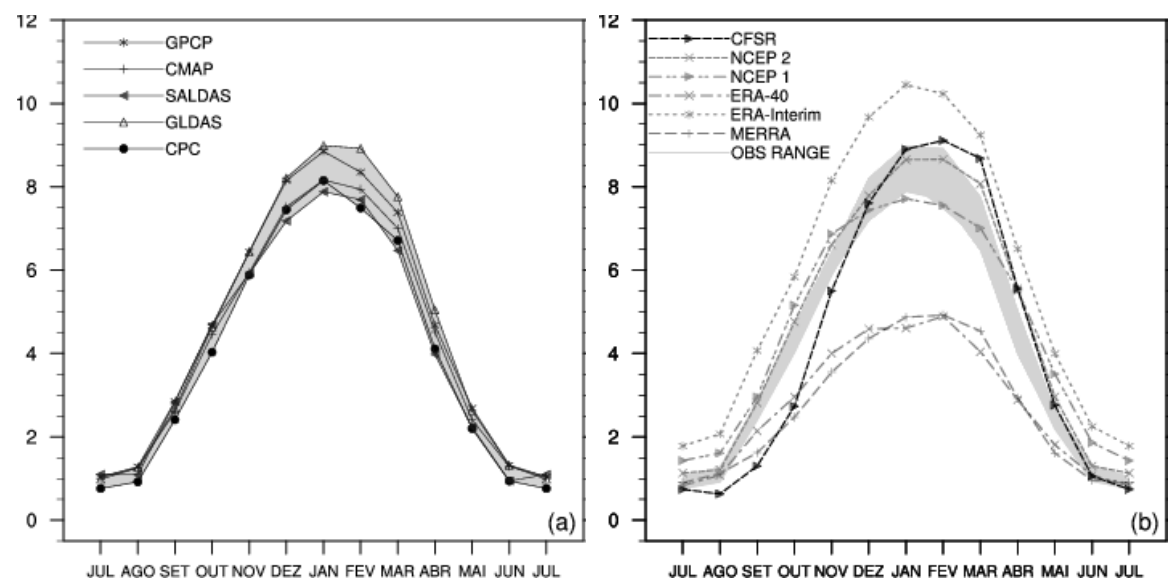

Figura 2 - Precipitação Climatológica (mm/dia) mensal dos produtos de observação CPC, GLDAS, SALDAS, CMAP e GPCP (a) e das reanálises MERRA, ERA-Interim, ERA-40, NCEP 1, NCEP 2 e NCEP CFSR (b) média para a região de estudo. 

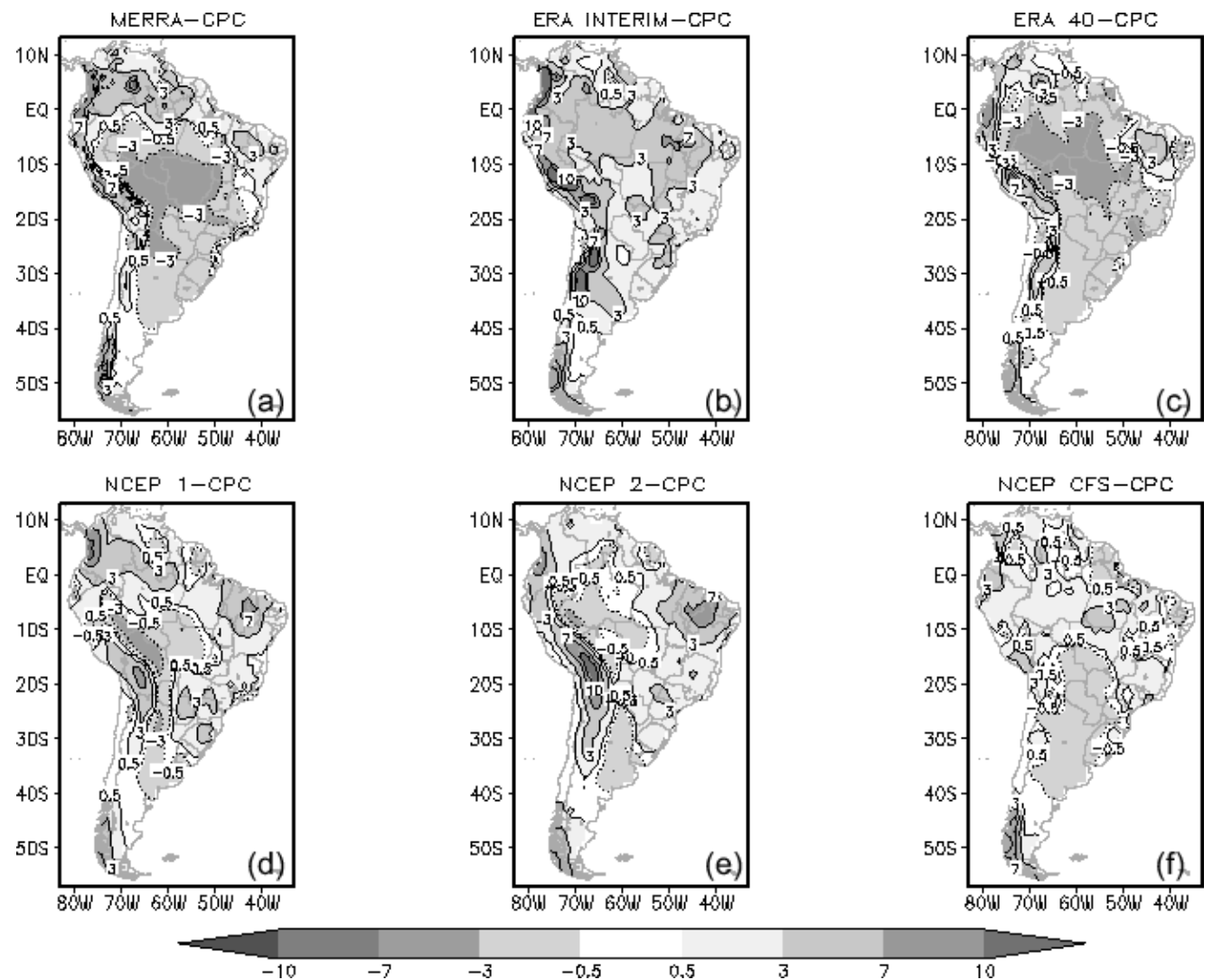

Figura 3 - Diferença entre a precipitação climatológica (mm/dia) entre as reanálises MERRA (a), ERA-Interim (b), ERA-40 (c),NCEP 1 (d), NCEP 2 (e) e NCEP CFSR (f) e o CPC, para os meses do verão (DJF) do HS. Linhas pontilhadas indicam valores negativos de desvios de precipitação.

máximo entre 2 e $4 \mathrm{~mm} /$ dia) para o período estudado. Este fato mostra um avanço dessa nova reanálise em relação às anteriores do NCEP.

Na Figura 6 os diagramas de Taylor apresentam o skill das reanálises de precipitação com relação ao conjunto de dados de referência (CPC). Durante os meses de verão para a região da ZCAS (Figura 6a), os produtos de precipitação observada (SALDAS, CMAP, GPCP e GLDAS) tendem a se agrupar próximos ao ponto de referência, mostrando valores de correlação entre 0,6 e 0,9. Em geral, as reanálises apresentam baixos valores de correlação, abaixo de 0,6. No entanto, é importante salientar que a reanálise CFSR (nova geração de reanálises), apresenta uma evolução em relação aos produtos antigos. Na região da ZCAS, o CFSR apresenta os melhores resultados em relação ao CPC, comparando-se ao SALDAS (produto de observação) em determinados anos. Analisando o desvio padrão das séries, as reanálises MERRA e ERA-Interim apresentam valores de STD próximos ao valor de REF (pouca variabilidade da série). Os maiores desvios são apresentados pelas reanálises NCEP 1 e ERA-40 com uma amplitude maior que a referência (CPC) e as reanálises NCEP 2 e CFSR que possuem uma variabilidade maior que o CPC. Em uma análise sazonal na região da ZCAS, no inverno austral (Figura 6b) a correlação espacial das reanálises é maior que nos meses verão. Este resultado é consistente com o observado em Bosilovich (2008) que indica que no período de verão do Hemisfério Sul (HS), as reanálises apresentam um erro sistemático na avaliação da precipitação sobre a região de interesse. No entanto, é importante ressaltar que em algumas partes da AS, como a região central do Brasil, há uma cobertura menor de registros de precipitação, o que dificulta as análises. Além disso, durante os períodos com redução da precipitação, aumenta significativamente a correlação entre os modelos.

Analisando o fornecimento de umidade para a atuação da ZCAS, a Figura 7 mostra o transporte de umidade tridimensional, a evaporação e a precipitação gerada através da reanálise MERRA sobre a América do Sul. Nos meses de verão do HS, é possível observar um fluxo de norte/nordeste que evidencia o transporte de umidade a partir da Amazônia, o qual se associa mais ao sul ao Jato de Baixos Níveis (JBN) a leste da Cordilheira dos Andes (Figura 7a). Este padrão configura uma predominância do transporte de umidade pelos ventos alísios para a região da ZCAS e, como consequência, uma forte convergência de umidade integrada na coluna na maior parte da região de estudos (valores negativos na Figura 7a). A evaporação gerada pela reanálise MERRA (Figura 7b) 

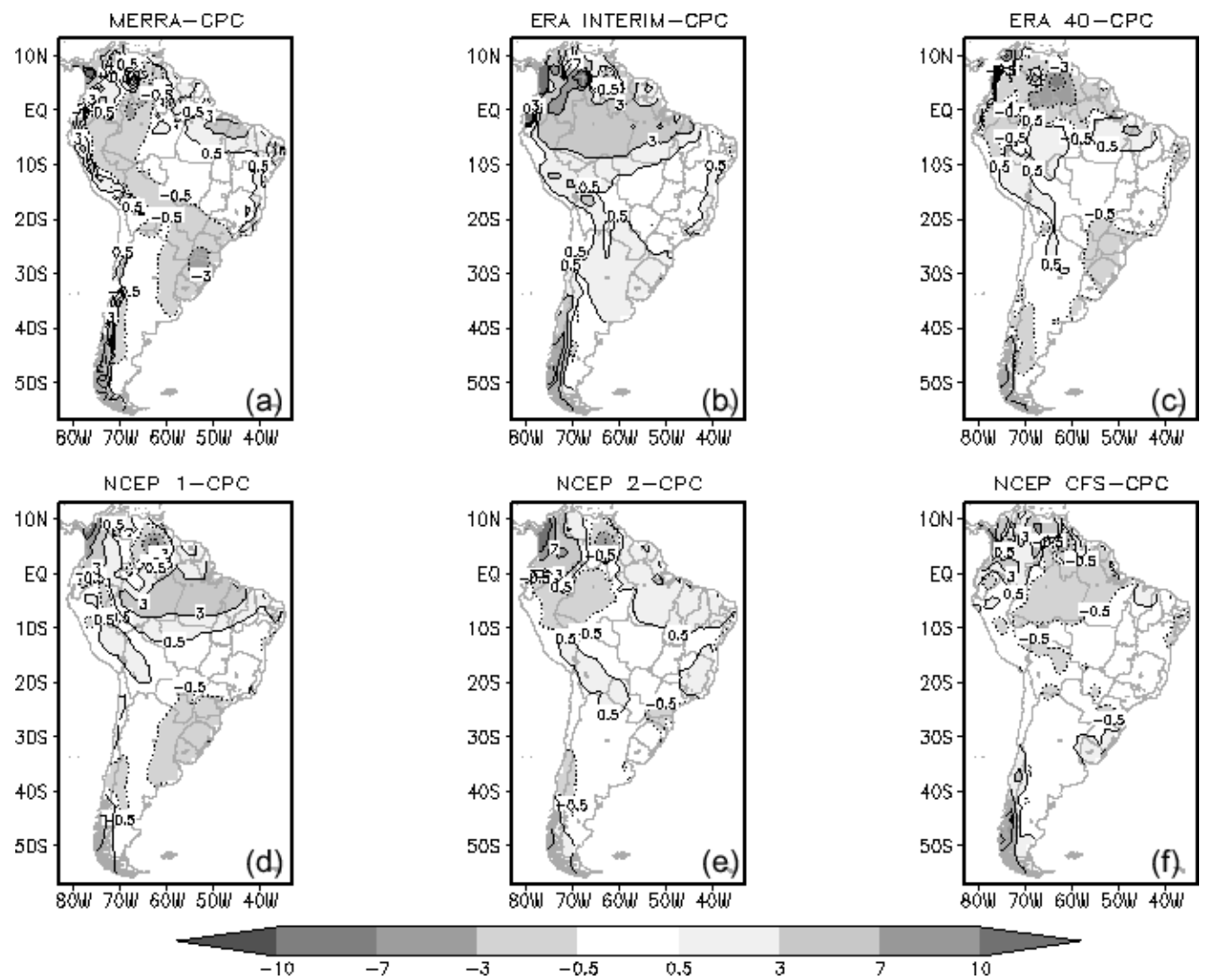

Figura 4 - Idem a Figura 3, para os meses do inverno (JJA) do HS.
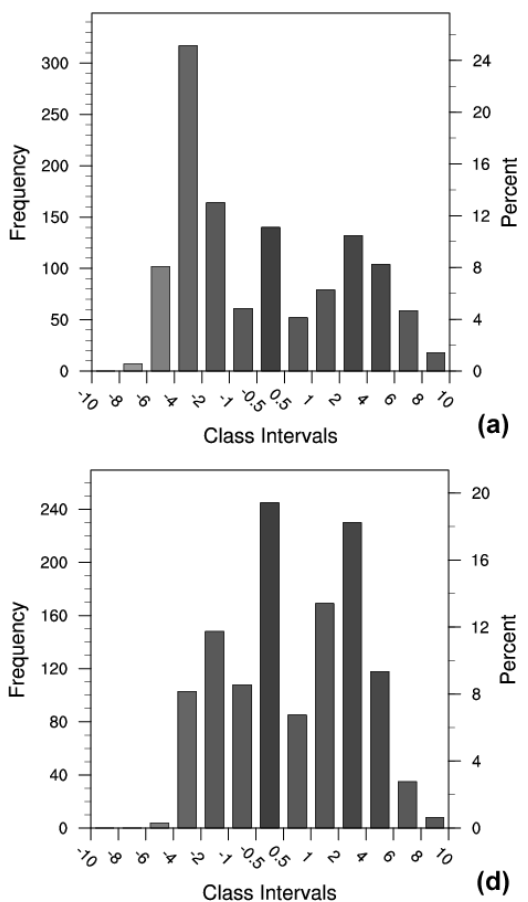
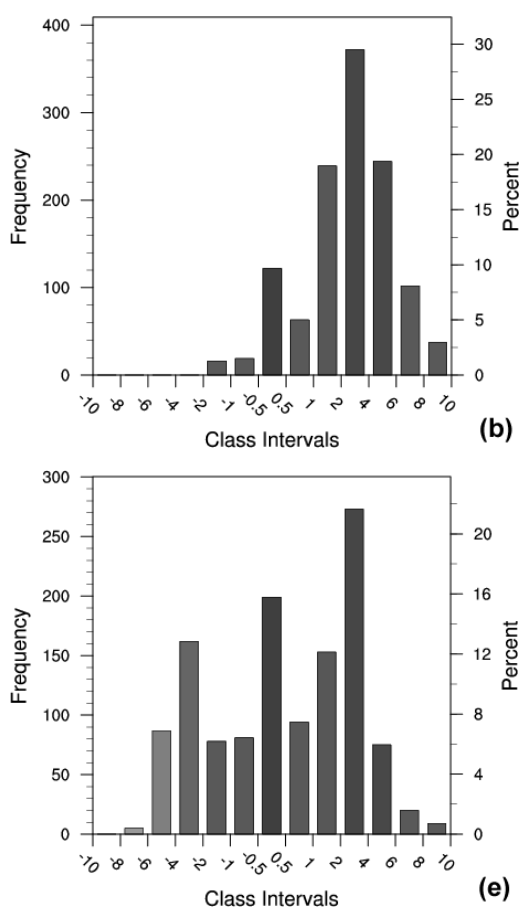
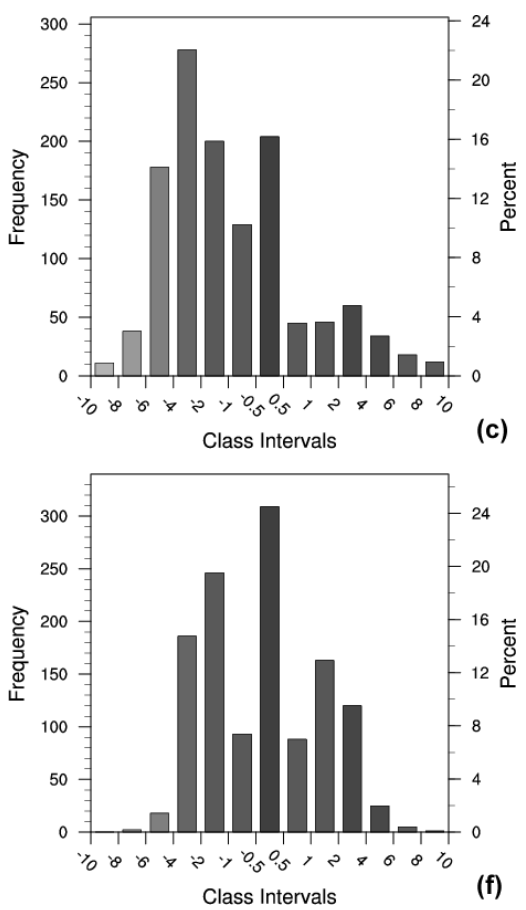

Figura 5 - Histogramas da diferença de precipitação ( $\mathrm{mm} /$ dia) média de verão do HS das reanálises MERRA (a), ERA-Interim (b), ERA-40 (c), NCEP 1 (d), NCEP 2 (e) e CFSR do NCEP (f) e a climatologia do CPC para a região da ZCAS. 

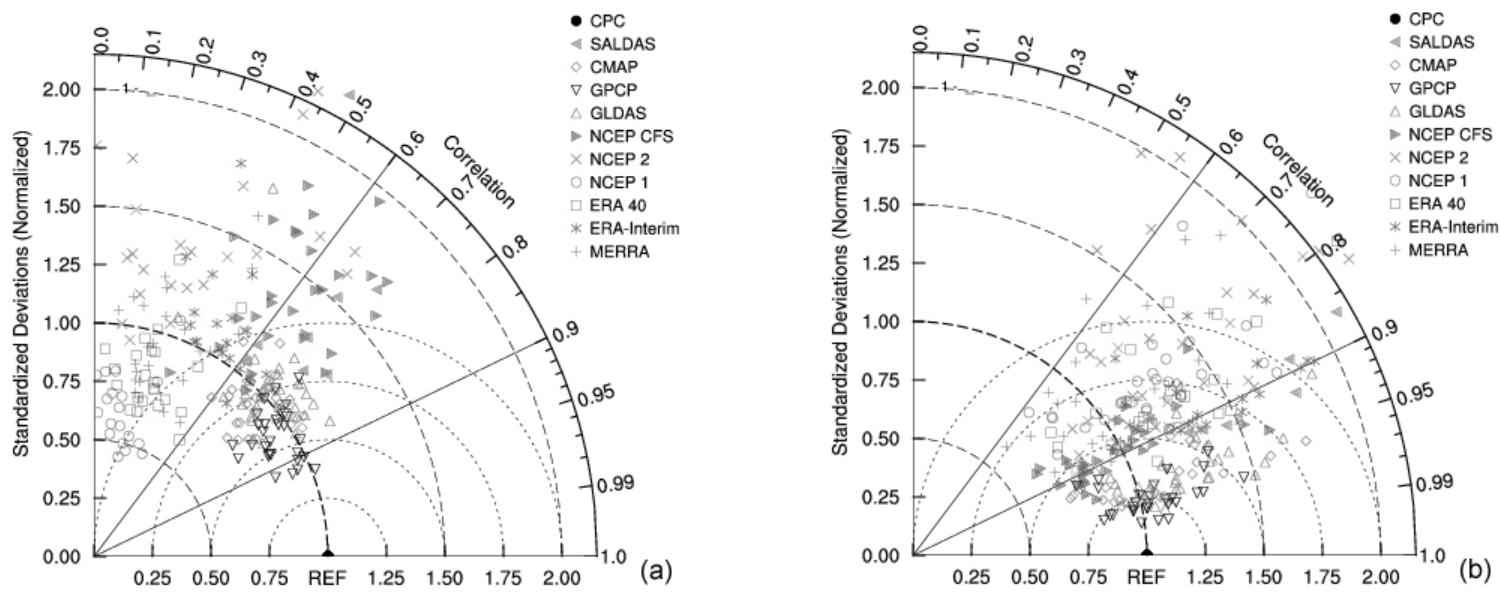

Figura 6 - Diagramas de Taylor para a correlação e desvio padrão médio na região da ZCAS para os meses de verão (a) e inverno (b) do HS, usando o CPC como referência.
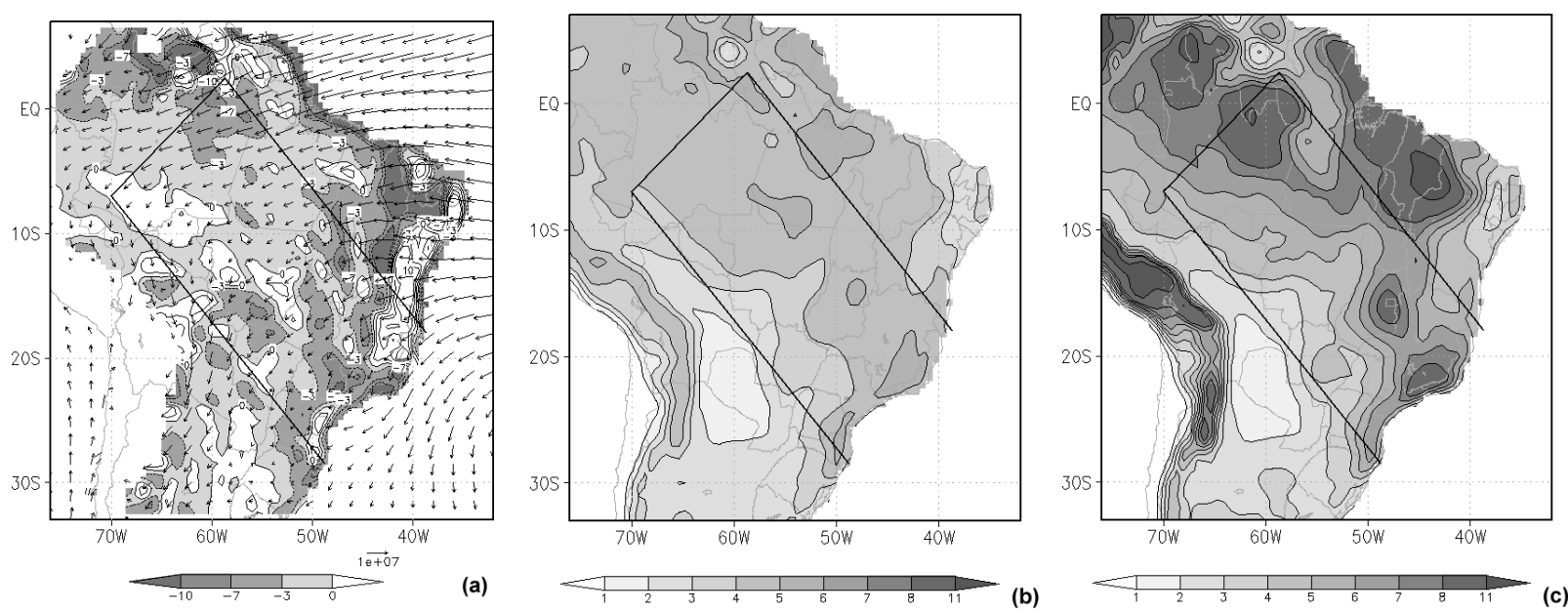

Figura 7 - Vetor fluxo integrado de umidade (Kg.m-1s-1) e divergência do fluxo integrado de umidade (mm.dia-1) médio na coluna atmosférica (valores negativos sombreados representam convergência) (a), evaporação (mm.dia-1) (b) e precipitação acumulada (mm.dia-1) médias (c) para a reanálise MERRA nos meses de verão (DJF).

contribui como uma fonte de umidade local para a ZCAS, em média, entre $2 \mathrm{~mm} /$ dia na borda sudoeste do sistema e 6 $\mathrm{mm} /$ dia no extremo nordeste. A parcela de umidade, que é convertida em precipitação (Figura 7c) na região da ZCAS, tem seu máximo deslocado para nordeste, com três núcleos intensos de precipitação sobre o norte do Estado do Amazonas, Goiás e o leste da Região Sudeste. Nesta região, sugere-se que as Serras do Mar e da Mantiqueira devem exercer um papel importante para a intensificação da precipitação orográfica durante os episódios de ZCAS, contribuindo para os máximos de precipitação (Grimm et al., 2007). Na região Amazônica, o núcleo máximo de precipitação está associado a processos termodinâmicos da atmosfera.

Como pela Figura 3a a reanálise MERRA mostra um desvio negativo de precipitação em relação ao CPC sobre grande parte da região da ZCAS continental, sugere-se que o modelo não representa bem o transporte de umidade para esta região mais ao sul, sendo que em alguns Estados da Região CentroOeste se verificam valores de divergência (positivos) de fluxo de umidade, que deve contribuir para o déficit de precipitação na região.

O transporte de umidade calculado pela reanálise do CFSR (Figura 8a) mostra alguns aspectos semelhantes ao calculado pelo MERRA, como o fluxo de umidade, através dos ventos alísios, para a região da ZCAS e a convergência de umidade nesta região. No entanto, ao contrário da reanálise MERRA, esta reanálise mostra valores maiores de convergência de umidade e de evaporação (Figura 8b) sobre a região. Como resultado, o campo de precipitação do CFSR mostra um padrão de chuvas consistente com a localização da ZCAS, com uma banda bem definida de precipitação localizada na região climatológica da ZCAS e consistente com os valores do CPC (Figura 3). 

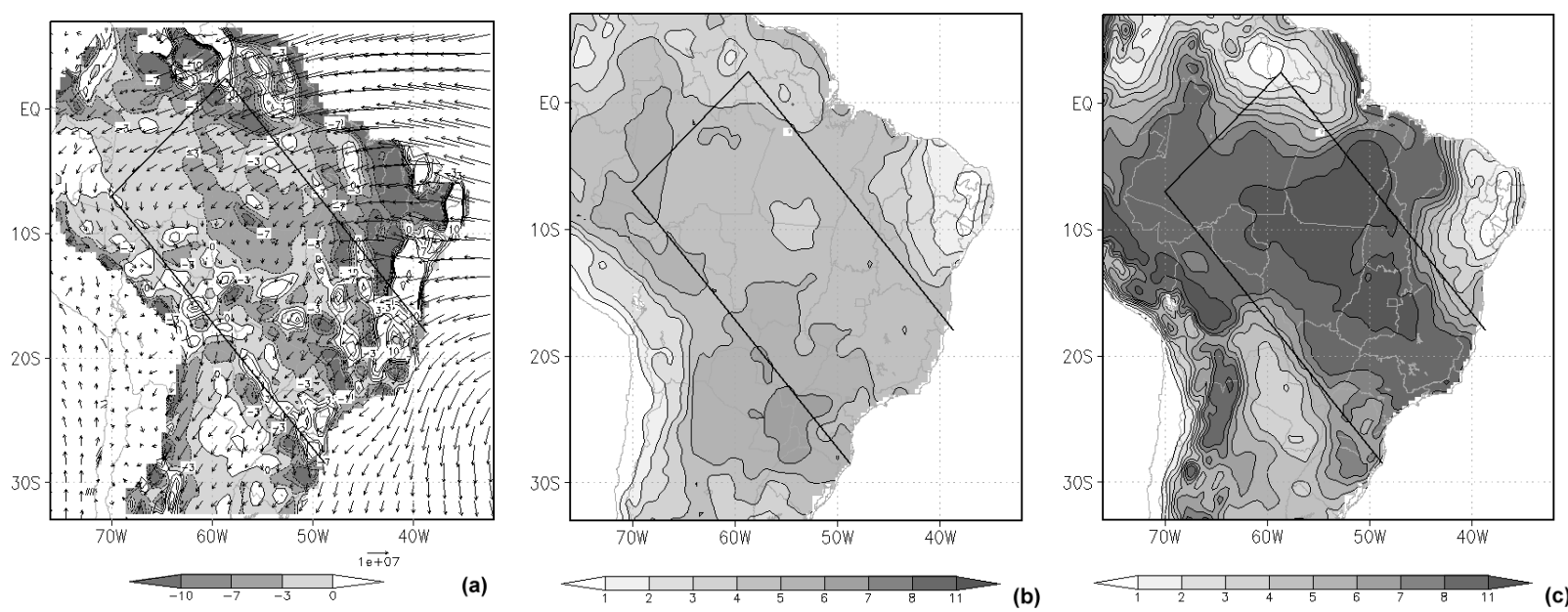

Figura 8 - Idem a Figura 7 para a reanálise CFSR do NCEP.

\section{CONCLUSÃO}

Como conclusão, este trabalho evidencia o avanço das novas reanálises na tentativa de representar de forma mais adequada a variável precipitação acumulada, que é um produto sensível tanto à assimilação das observações no sistema de reanálise, como às parametrizações do modelo. Especificamente, alguns resultados podem ser evidenciados. Todas as reanálises representam bem o ciclo anual dos produtos de precipitação, sendo que as reanálises do NCEP são as que mais se aproximam das curvas de precipitação observada. Na região central da AS, as reanálises do MERRA/ ERA-40 e do ERA-Interim apresentam sinais opostos nas diferenças em relação ao $\mathrm{CPC}$ (evidenciado também nos histogramas). Já o NCEP CFSR é a reanálise que mostra o menor viés para todo o continente, incluindo a região dos Andes. Acredita-se que ao melhor desempenho dessa nova geração de reanálise deve-se ao fato de utilizar um modelo acoplado oceano-atmosfera, além da alta resolução espacial do modelo e também a assimilação de radiâncias. Os diagramas de Taylor mostram que somente a reanálise do NCEP CFSR possui correlações próximas as dos produtos de precipitação. As outras apresentam correlações menores, abaixo de 0,6. Além disso, algumas reanálises (MERRA e ERA-Interim) apresentam erros sistemáticos (viés) em algumas partes do continente, com baixa densidade de pluviômetros. O Oceano Atlântico é a fonte principal do fluxo de umidade para a ZCAS, ressaltado principalmente pela reanálise CFSR do NCEP, pois a evaporação local é insuficiente para fornecer toda umidade para o total de precipitação gerada na região da ZCAS. Na região SE do Brasil, acredita-se que a topografia possa ter um papel importante para a convergência de umidade. Já na parte noroeste da ZCAS, este fator deve estar associado a processos termodinâmicos.

\section{AGRADECIMENTOS}

Os autores agradecem o suporte financeiro recebido do Inter-American Institute for Global Change Research (IAI) Cooperative Research Network 2094 (CRN-2094), a Coordenação de Aperfeiçoamento de Pessoal de Nível Superior pela Coordenação de Programas de Qualificação de Quadros Docentes (PICDTec/CAPES) pela bolsa de estudos e a Ernesto Hugo Berbery pela significativa contribuição ao trabalho. Esta pesquisa foi financiada parcialmente pelo Terrestrial Hydrology Program (NNX08AE50G) da NASA (National Aeronautics and Space Administration).

\section{REFERÊNCIAS BIBLIOGRÁFICAS}

ADLER, R.F. and Coauthors: The version-2 Global Precipitation Climatology Project (GPCP) monthly precipitation analysis (1979-present). Journal of Hydrometeorology, 4, 11471167, 2003.

BOSILOVICH, M. NASA's Modern Era Retrospectiveanalysis for Research and Applications: Integrating Earth Observations. Earthzine. Disponivel em http://www. earthzine.org/2008/09/26/nasas-modern-era-retrospectiveanalysis/, 2008.

CARVALHO, L. M. V.; JONES C.; LIEBMANN, B. The South Atlantic Convergence Zone: intensity, form, persistence, and relationships with intraseasonal to interannual activity and extreme rainfall. Journal of Climate, v. 17, p. 88-108, 2004.

CASARIN, D. P.;. KOUSKY, V.E. Precipitation anomalies in the southern part of Brazil and variations of the atmospheric circulation, Revista Brasileira de Meteorologia, 1, pp. 83-90, 1986.

CHAVES, R R.; SATYAMURTY, P. Estudo das Condicões Regionais Associadas a um Evento de Forte ZCAS em 
Janeiro de 2003. Revista Brasileira de Meteorologia, v.19, n.1, 89-98, 2006.

DRUMOND, A.; NIETO, R; GIMENO, L.; AMBRIZZI, T. A Lagrangian identification of major sources of moisture over Central Brazil and La Plata Basin, Journal of Geophysical. Research, 113, D14128, doi:10.1029/2007JD009547, 2008.

FERREIRA, N.J.; SANCHES, M.; SILVA DIAS, M.A.F. Composição da Zona de Convergência do Atlântico Sul em Períodos de El Niño e La Niña. Revista Brasileira de Meteorologia, v.19, n.1, 89-98, 2004.

FIGUEROA, S. N.; NOBRE, C. Precipitation distribution over central and western tropical South America. Climanálise, v. 5, p. 36-45, 1990.

GAN M.A., KOUSKY V.E., ROPELEWSKI C.F. The South America Monsoon Circulation and Its Relationship to Rainfall over West-Central Brazil. Journal of Climate 17(1): 47-66, 2004.

GANDIM, L. S. Objective Analysis of Meteorological Fields. Israel Program for Scientific Translation, Jerusalem, 1965. 242 p.

GIBSON, J.K., P. KALLBERG, S. UPPALA, A. HERNANDEZ, A. NOMURA; E. SERRANO. ERA Description. ECMWF Re-Analysis Project Report Series, 1. 1997.

GONÇALVES, L.G.G and Coauthors. The South American Land Data Assimilation System (SALDAS) 5-Yr Retrospective Atmospheric Forcing Datasets. Journal of Hydrometeorology, 2009; 10: 999-1010.

GRIFFIES, M.J.; HARRISON, R. C. PACANOWSKI; ROSATI, A. Technical guide to MOM4. GFDL Ocean Group. Technical Report No. 5, 337 pp. Disponível em www.gfdl. noaa.gov/ fms, 2004.

GRIMM, A. M. Interannual climate variability in South America: impacts on seasonal precipitation, extreme events and possible effects of climate change. Stochastic Environmental Research and Risk Assessment. v. 25, n. 4, p. 537-554, DOI: 10.1007/s00477-010-0420-1, 2011.

GRIMM, A. M.; ZILLI, M. T. Interannual variability and seasonal evolution of summer monsoon rainfall in South America. Journal of Climate, v. 22, p. 2257-2275, 2009.

GRIMM, A. M.; J. PAL; F. GIORGI, Connection between spring conditions and peak summer monsoon rainfall in South America: Role of soil moisture, surface temperature, and topography in eastern Brazil. Journal of Climate, v. 20, p. 5929-5945, 2007.

GRIMM, A. M.; SILVA DIAS, P. L. Analysis of tropicalextratropical interactions with influence functions of a barotropic model. Journal of the Atmospheric Sciences, 52, 3538-3555, 1995.

HERDIES, D. L.; DA SILVA A. ; SILVA DIAS M.A.F. ; FERREIRAR.N. The moisture budget of the bimodal pattern of the summer circulation over South America. Journal of Geophysical Research, Washington-DC, v. 107, n. D20, p. 42-1-42-10, 2002.

JANOWIAK, J. E., GRUBER, A.; KONDRAGUNTA, C.R.; LIVEZEY, R.E.; HUFFMAN, G.F. A comparison of the NCEP-NCAR reanalysis precipitation and the GPCP rain gauge-satellite combined dataset with observational error considerations. Journal of Climate, 11, 2960-2979.1998.

JONES, C.; CARVALHO, L. M. V. Active and break phases in the South American Monsoon System. Journal of Climate, v.15, p.905-914, 2002.

JONES, C.; HOREL, J. D. A circulação da Alta da Bolívia e a atividade convectiva sobre a América do Sul. Revista Brasileira de Meteorologia, v. 5, p. 379-387, jun. 1990.

KALNAY. E. and Coauthors. The NCEP/NCAR 40-year reanalysis project. Bulletin of the American Meteorological Society, 77, 437-471, 1996.

KANAMITSU, M. and Coauthors. NCEP-DOE AMIP-II Reanalysis (R-2). Bulletin of the American Meteorological Society, 83, 1631-1643. 2002.

LIN, J.-L. The Double-ITCZ Problem in IPCC AR4 Coupled GCMs: Ocean-Atmosphere Feedback Analysis. Journal of Climate, v.20, p.4497-4525, 2007.

MARENGO, J. A. The characteristics and variability of the atmospheric water balance in the Amazon basin: Spatial and temporal variability. Climate Dynamics, v.24, p.1122, 2005.

MARTON, E. Oscilações intrasazonais associadas a Zona de Convergência do Atlântico Sul no Sudeste Brasileiro. Tese (Doutorado em Meteorologia) - Instituto Astronômico e Geofísico, Universidade de São Paulo, São Paulo, 2000.

MENDONÇA, R. W.B. Estudo das trocas horizontais e verticais durante episódios de ZCAS: influência da resolução das análises e dos modelos e de parametrização de convecção. Tese de Doutorado em Meteorologia. INPE, 2005. 204 p.; - (INPE-14213-TDI/1114)

NOBRE, C. Ainda sobre a Zona de Convergência do Atlântico Sul: a importância do Oceano Atlântico. Climanálise, v. 3, n. 4, p. 30-35, abr. 1988.

NOGUES-PAEGLE J.and Coauthors. Progress in Pan American CLIVAR Research: Understanding the South American Monsoon, Meteorologica. 1and 2. 3-32, 2002.

ONOGI, K. and Coauthors. The JRA-25 Reanalysis. Journal of the Meteorological Society of Japan, 85, 3, 369-432. 2007.

PAOLINO, D., Q. YANG, B. DOTY, J. KINTER, J. SHUKLA; D.M. STRAUS. A Pilot Reanalysis Project at COLA. Bulletin of the American Meteorological Society, 76, 697-710. 1995.

PEZZI , L.P. ; CAVALCANTI, I.F.A. Testes de sensibilidade com o MCGA CPTEC/COLA usando dois esquemas 
diferentes de convecção. Anais do XI Congresso Brasileiro de Meteorologia, 2000.

RODELL, M. and Coauthors. The Global Land Data Assimilation System. Bulletin of the American Meteorological Society, 85(3):381-394. 2004.

SAHA, S. et al. The NCEP Climate Forecast System Reanalysis. Submitted to the Bulletin of the American Meteorological Society. Revised: March 25, 2010

SATYAMURTI, P.; RAO, V. B. Zona de Convergência do Atlântico Sul. Climanálise, v. 3, p. 31-35, mar. 1988.

SCHUBERT, S. D.; MIN, W.; TAKACS, L.; Joiner, J. Reanalysis of historical observations and its role in the development of the Goddard EOS Climate Data Assimilation System. Advances in Space Research, 19, Issue 3, 1997, Pages 491-501.

SILVA, V.B.S; KOUSKY, V.E.; HIGGINS, R.W. Daily Precipitation Statistics for South America: An Intercomparison between NCEP Reanalyses and Observations Journal of Hydrometeorology, 12, 101-117, 2011

SILVA, A.E. Variabilidade da Circulação e Umidade no Regime de Monção da America do Sul. Tese (Doutorado em Meteorologia) - Instituto Astronômico e Geofísico, Universidade de São Paulo, São Paulo, 2009. Disponível em $<$ http://www.iag.usp.br/pos/sites/default/files/d_ana_e_ silva.pdf $>$.
SILVA DIAS, P. L. and Coauthors. As chuvas de março de 1991 na região de São Paulo. Climanálise, v. 6, p. 44-59, maio 1991.

SIMMMONS, A., S. UPPALA, D. Dee, S. KOBAYASHI. ERA-Interim: New ECMWF reanalysis products from 1989 onwards. ECMWF NEWSLETTER 110: 29, 2007.

TAYLOR, K. E.: Summarizing multiple aspects of model performance in a single diagram. Journal of Geophysical Research, 106, 7183-7192, 2001.

UPPALA, S. M. and Coauthors. The ERA-40 reanalysis Submetido ao Quarterly Journal of the Royal Meteorological Society, 2005.

XIE, P.; CHEN, M.; SHI, W;. CPC unified gauge analysis of global daily precipitation. To be submitted to Journal of Hydrometeoroly 2010.

XIE, P.; ARKIN, P.A. Global precipitation: A 17-year monthly analysis based on gauge observations, satellite estimates, and numerical model outputs. Bulletin of the American Meteorological Society, 78, 2539 - 2558. 1997.

ZHOU , J. e LAU, K.M. Does monsoon climate exist over South America? Journal of Climate, v. 11, p. 1020-1040, 1998. 